\title{
Les paradis de papier : les sites balnéaires bretons magnifiés par les guides (1860-1939)
}

Paper paradise: how tourist guides idealised seaside towns in Brittany

(1860-1939)

Johan Vincent

\section{OpenEdition}

\section{Journals}

Édition électronique

URL : http://journals.openedition.org/abpo/2496

DOI : $10.4000 /$ abpo.2496

ISBN : 978-2-7535-2236-7

ISSN : 2108-6443

\section{Éditeur}

Presses universitaires de Rennes

\section{Édition imprimée}

Date de publication : 31 décembre 2012

Pagination : 99-112

ISBN : 978-2-7535-2234-3

ISSN : 0399-0826

\section{Référence électronique}

Johan Vincent, «Les paradis de papier : les sites balnéaires bretons magnifiés par les guides

(1860-1939) ", Annales de Bretagne et des Pays de l'Ouest [En ligne], 119-4 | 2012, mis en ligne le 31

décembre 2014, consulté le 02 mai 2019. URL : http://journals.openedition.org/abpo/2496 ; DOI

$10.4000 / a b p o .2496$ 


\title{
Les paradis de papier : les sites balnéaires bretons magnifiés par les guides (1860-1939)
}

\author{
Johan VINCENT \\ Historien public, chercheur associé au CERHIO, UMR 6258
}

Les années 1860 correspondent en France à la période d'essor des guides touristiques d'audience nationale. Le premier guide Joanne portant sur la Bretagne date de 1863. Il ne porte que sur la partie gallo (partie est de la Bretagne) tandis que le premier volume couvrant toute la région paraît en 1867 (Itinéraire de la France : Bretagne). Après Hachette, d'autres éditeurs nationaux ou internationaux investissent ce territoire : Bædeker (pour une zone géographique plus large), Touring-Club de France, Michelin. Certains ne publient qu'un seul et unique guide. Ils sont concurrencés, à l'échelle plus locale, par des guides d'abord écrits par des érudits locaux puis, à mesure que l'activité balnéaire se professionnalise, par les offices de tourisme créés en Bretagne à partir des années 1900. Au moment où les lignes ferroviaires atteignent les littoraux (si on excepte Saint-Nazaire, SaintMalo est la première station balnéaire bretonne à avoir sa gare, en 1864) et les lotissements des promoteurs colonisent les côtes, dans les années 1870-1880, le guide est idéal pour aider le curieux à trouver sa destination de voyage.

Les guides sont un matériau régulièrement exploité par les historiens du tourisme, et plusieurs d'entre eux - Dominique Rouillard ${ }^{1}$, Catherine Bertho-Lavenir ${ }^{2}$, Bernard Toulier ${ }^{3}$ - ont observé que les promoteurs des stations touristiques, tout comme les guides, ont contribué à créer un paysage de bord de mer imaginaire séduisant. L'autorité morale que les édi-

1. RoUlLLARD, Dominique, Le Site balnéaire, Liège, Pierre Mardaga, 1984.

2. BERTHO-LAVENIR, Catherine, La Roue et le stylo; Comment nous sommes devenus touristes, Paris : Odile Jacob, coll. « Le champ médiologique ", 1999, p. 24.

3. TOULIER, Bernard, "L'influence des guides touristiques dans la représentation et la construction de l'espace balnéaire (1850-1950) ", dans : ChABAUD, Gilles, et alii, Les Guides imprimés du XVI ${ }^{e}$ au XX $X^{e}$ siècle; Villes, paysages, voyages, Paris, Belin, coll. « Mappemonde ", 2000 . 
teurs s'accordent ${ }^{4}$ et l'état d'esprit de certains guides, comme les guides Michelin étudiés par Marc Francon ${ }^{5}$, ont été pour partie étudiés mais une analyse fine du message et de son évolution reste à produire. Alors qu'il paraîtrait logique que l'attirance pour le site soit une des motivations principales du touriste, l'étude des guides révèle l'élaboration d'un discours, plus ou moins général, sur les attraits des lieux et des paysages. Catherine Bertho-Lavenir explique même que, dès les années 1860, la Bretagne des guides est formée d'itinéraires jalonnés de lieux consacrés et de stations balnéaires : "Coupé de la Bretagne réelle, le touriste prend l'habitude d'en consommer des ersatz ${ }^{6}$." Or, avec les guides, nous serions bien en phase avec le mythe décrit par Roland Barthes. "Le mythe ne nie pas les choses, sa fonction est au contraire d'en parler : simplement il les purifie, il les innocente, les fonde en nature et en éternité [...]. Les choses ont l'air de signifier toutes seules ${ }^{7}$. "Comment a été créée, pour les touristes, cette sorte de mythe sur le littoral breton?

Le site, défini par Pierre George et Fernand Verger comme " un ensemble de lieux sur lesquels règnent des conditions écologiques similaires du point de vue forestier ${ }^{8}$ ", par Yves Lacoste comme "le cadre topographique de l'emplacement d'une ville ${ }^{9}$ ", permet à toutes les stations balnéaires d'être représentées : petites comme grandes, elles possèdent toutes un cadre naturel. Selon quels principes les éditeurs de guide procèdent-ils pour privilégier certaines stations balnéaires et finalement créer des destinations idéales? L'analyse des guides sur le long terme sur quasiment un siècle dans cet article - permet de s'apercevoir que c'est la station balnéaire qui tend à mettre en valeur le site. L'étude d'un corpus d'une centaine de guides sur la Bretagne révèle une mentalité qui imprègne progressivement le discours, assimilée dans des thématiques régionales qui s'affirment au cours du siècle, et qui conduira à l'acceptation d'un réaménagement des sites eux-mêmes. Après avoir montré que le premier objet des guides est de fixer l'intérêt du site, en particulier dans les premières années d'édition, nous analyserons comment le discours pour y parvenir a été construit à partir d'une grille référentielle de lecture du territoire. Ce discours est à la base, au xx siècle, d'un cercle vertueux (ou vicieux) du succès balnéaire.

4. GRITTI, Jules, "Les contenus culturels du Guide bleu », Communications n 10, 1967, p. 63-64.

5. Francon, Marc, Le Guide Vert Michelin; L'invention du tourisme culturel populaire, Paris : Éditions Economica, coll. "Économies et sociétés contemporaires ", 2001, p. 6.

6. Bertho-LaVEniR, Catherine, "L'invention de la Bretagne : genèse sociale d'un stéréotype ", Actes de la recherche en sciences sociales $n^{\circ} 35,1980$, p. 61.

7. BARTHEs, Roland, Mythologies, Paris, Seuil, 1957, p. 230-231.

8. George, Pierre, et Verger, Fernand (dir.), Dictionnaire de la géographie, Paris, PUF, 2009, p. 405.

9. LACOSTE, Yves, De la géopolitique aux paysages : dictionnaire de la géographie, Paris, Armand Colin/VUEF, 2003, p. 351. 


\section{Un contexte local porteur de différenciations}

L'objectif des guides est de démontrer l'intérêt du site plus que de dépeindre la réalité. Apportant la preuve d'une certaine reconnaissance des stations balnéaires citées, les éditeurs et les auteurs de guide ne sont pourtant pas si désintéressés.

\section{La présence humaine, créateur d'intérêt touristique}

L'arrivée de touristes n'est possible, généralement, que dans les sites aménagés qui permettent à tous d'en tirer profit. Les premiers guides sur la Bretagne à diffusion nationale commencent donc par rassurer le touriste. Au milieu du XIX ${ }^{\mathrm{e}}$ siècle, l'affluence balnéaire est un signe favorable. Le Pouliguen est " particulièrement fréquenté par les familles qu'effarouchent le luxe et l'agitation mondaine du Croisic ${ }^{10}$ ". D'ailleurs, sans agglomération sociale sur le bord de mer, la plage n'existe pas dans le guide : ainsi, Adolphe Joanne, en suivant la route côtière entre Pléneuf et Matignon, ne signale en 1867 aucune plage, ni même la mer (alors qu'il décrit le port de Dahouet), parce que Val-André et Sables-d'Or-les-Pins n'existent pas à l'époque ${ }^{11}$. Au milieu du XIX ${ }^{\mathrm{e}}$ siècle, les autochtones sont encore évoqués, car les baigneurs auront à les côtoyer (pour se loger par exemple) - une description qui aura disparu dans certains guides dès le début du $\mathrm{xx}^{\mathrm{e}}$ siècle, ou qui sera effectuée dans une introduction générale (sous forme de types, déjà évoqués par Roland Barthes pour le Guide bleu). Adolphe Joanne explique le succès du Croisic en 1867, "à l'extrémité d'une péninsule dont les habitants, leurs mœurs et leur industrie offrent un vif intérêt ${ }^{12}$ ". L'appréhension des sites pittoresques sans la présence d'habitants, à partir de la fin du XIX ${ }^{\mathrm{e}}$ siècle, est sans doute due à une pratique toujours plus individualisée du tourisme et à une professionnalisation alors affirmée de l'activité balnéaire : l'appropriation se fait sans élément perturbateur. C'est l'avènement du pays sage (sage dans le sens de docile). Les guides adoptent alors un discours ambivalent : l'attraction des sites, consacrée en fait par la présence humaine, est maintenant présentée à l'aune des paysages. Ce sont ainsi les paysages qui commencent à attirer les baigneurs à Damgan dans les années $1900^{13}$. Sur les clichés illustrant la revue du Touring-Club de France au xx ${ }^{\mathrm{e}}$ siècle, Catherine Bertho-Lavenir constate la marginalité, proche de l'absence, des autochtones et des touristes ${ }^{14}$. L'élaboration des processus de classement de monuments naturels (1906) et culturels (1913) est également révélatrice de cette nouvelle sensibilité.

10. Joanne, Adolphe, Itinéraire général de la France : Bretagne, Paris, Hachette, coll. "Guides Joanne ", 1867, p. 532.

11. Ibidem, p. 410-411.

12. Ibid., p. 536.

13. ARdouin-Dumazet, Voyage en France (4e partie); Littoral breton de l'Atlantique, Paris/ Nancy, Berger-Levrault et Cie, 1909, p. 159.

14. BERTHO-LAVENIR, Catherine, op. cit., p. 274 et p. 285. 
L'étude du discours touristique permet de constater une sensibilité plus forte aux évolutions balnéaires qu'au paysage primitif de l'installation. En fait, les sites " sauvages " sont plus souvent mis en avant dans les récits de voyage. Dans les guides, l'intérêt pour les populations au milieu du XIX ${ }^{\mathrm{e}}$ siècle compense la nature du littoral français, à l'époque ressentie par les visiteurs comme hostile. Il faut remarquer qu'en France, le villégiateur ne construit pas de villas sur le bord de mer avant les années 1840 et même avant les années 1850 pour la Bretagne. La colonisation touristique des abords de la baie du Pouliguen, que l'on peut suivre d'une édition de guide à l'autre, est caractéristique du glissement géographique de l'intérêt du site. La plage de La Baule-Escoublac, avant la fixation du sable par les plantations de pins, est présentée comme une étendue de dunes qui «éveille dans l'âme les impressions les plus tristes. C'est en petit, l'image complète du désert, avec son silence, sa solitude et sa stérilité ${ }^{15}$ ". En 1863, Pol de Courcy préfère la présence humaine et conseille la plage voisine du Pouliguen, selon lui une des plus belles de la côte ${ }^{16}$. Adolphe Joanne, dans son guide paru en 1867, reprend mot pour mot cette invitation à choisir Le Pouliguen. Dans l'édition Joanne de 1873 apparaît sur l'autre pointe qui clôt la baie, Pornichet, " petite station de bains de $\operatorname{mer}^{17}$ ", sans plus de qualificatif. En 1885, Paul Joanne évoque Pornichet, " station de bains de mer très fréquentée " avec sa " belle plage de sable fin ", Escoublac-la-Bôle, "station balnéaire de création récente " et Le Pouliguen, avec " sa belle plage, en plein sud, bordée de charmantes villas ${ }^{18}$ ". La fin des désagréments, avec la plantation des pins au cours de la seconde moitié du XIX ${ }^{\mathrm{e}}$ siècle et l'édification des villas, permet enfin de profiter pleinement du site. Au début du Xx ${ }^{\mathrm{e}}$ siècle, la beauté du panorama est attribuée de manière équivalente, selon les auteurs, à l'une des trois stations. Adrien de Baroncelli en gratifie la plage du Pouliguen ${ }^{19}$; Paul Joanne celle de Pornichet ${ }^{20}$; l'auteur de $\grave{A}$ la France celle de La Baule (selon lui, une des plus belles plages qui soient au monde) ${ }^{21}$. C'est la présence de l'homme qui est à la base de l'intérêt au site et non le paysage.

\section{La prime aux grandes stations}

Dans ces conditions, les guides tendent à encenser les stations les mieux développées. À partir du moment où la station balnéaire est recon-

15. Pol DE COURCY, De Nantes à Lorient, à Saint-Nazaire et à Rennes, Paris, Hachette, coll. "Guides Joanne ", 1863, p. 187.

16. Ibidem, p. 186.

17. JoAnne, Adolphe, Itinéraire général de la France : Bretagne, Paris, Hachette, coll. "Guides Joanne ", 1873, p. 547.

18. JoAnne, Paul, Bretagne, Paris, Hachette, coll. « Guides Joanne-Guides Diamant », 1885, p. 292-293.

19. BARONCELLI, Adrien DE, Guides vélocipédiques : La Bretagne, plages bretonnes, Paris, 4e éd., sans date (années 1900), p. 118

20. JoAnne, Paul, Itinéraire général de la France : La Loire, Paris, Hachette, coll. « Guides Joanne ", 1900, p. 309.

21. Anonyme, À la France; Sites et monuments : Basse-Loire, Paris, Touring-Club de France, 1903, p. 95. 
nue, le site, et notamment la plage, gagne en qualité. Le sociologue Rachid Amirou explique que tout site ne peut devenir une destination touristique sans une consécration : la caution et l'adhésion mentale de la foule de consommateurs ${ }^{22}$. C'est en quelque sorte la célébrité décrite par Daniel Boorstin $^{23}$ : une personne ou un lieu connu pour être connu. En 1914, l'auteur du guide Bains de mer de Bretagne fait la distinction entre les plages qu'il juge digne d'intérêt. La station balnéaire peut être simple, avec une plage qui l'est tout autant : "Larmor[-Plage...] est une petite station balnéaire régionale, fréquentée surtout par les Lorientais; elle a une plage de sable, avec cabines. "Elle peut être plus importante : Quiberon, une des stations balnéaires les plus fréquentées, profite d'une belle plage tandis qu'à Sainte-Marguerite (Pornichet), petite station aristocratique, " la plage, légèrement inclinée mais sûre, est d'un beau sable fin ". Par contre, les communes littorales où l'activité balnéaire est marginale, peuvent être sujettes aux critiques : La Turballe, important port de pêche sardinier, décrit comme sale et populeux, ne dispose " que de l'immense grève de sable qui s'étend vers la gauche, jusqu'en face du Croisic, durant cinq kilomètres ${ }^{24}$ ". À Camaret-sur-Mer, " très petite station balnéaire ", on peut se baigner à une petite plage voisine du port " mais (recommandation importante) partout ailleurs sur les côtes environnantes, même sur les plus belles grèves, il faut se défier des courants marins qui sont des plus dangereux ${ }^{25} "$.

Ces consécrations sont renforcées par une rédaction des guides nullement déconnectée des réalités économiques. La puissance économique des grandes stations balnéaires influence le contenu car les guides sont en partie financés par elles. En effet, les conseils municipaux sont régulièrement sollicités pour subventionner les éditions. En 1903, faute d'argent, le conseil municipal de Pornichet ne donne pas suite aux " demandes présentées par divers éditeurs de guides de bain de mer tendant à obtenir des subventions de la commune pour faire de la publicité en faveur de Pornichet ${ }^{26}$ ". Ces demandes de subventions accordent un droit de regard sur les propos des guides sur la commune. Port-Louis accorde en 1896 vingt francs à l'éditeur La Fare (Les petits trous pas chers; Guide des familles aux bains de mer) pour être décrite comme une station balnéaire (ce qu'elle est alors depuis une cinquantaine d'années) et attirer ainsi les baigneurs. La subvention est annuellement attribuée (avec quelques diminutions) jusqu'en 1914. En 1905, le conseil municipal précise que « des erreurs ayant été constatées dans la notice parue l'année dernière, le maire est prié de les

22. AmIRou, Rachid, Imaginaire touristique et sociabilités du voyage, Paris, PUF, coll. « Le Sociologue ", 1995, p. 108.

23. Boorstin, Daniel, L'Image, Paris, Julliard, 1963.

24. Anonyme, Bains de mer de Bretagne, du Mont Saint-Michel à Saint-Nazaire, Paris, Hachette, coll. "Guides Joanne », 1914, p. 178, p. 191, p. 218 et p. 208.

25. Gruyer, Paul, Bains de mer de Bretagne, du Mont Saint-Michel à Saint-Nazaire, Paris, Hachette, coll. "Guides Joanne ", 1911, p. 133.

26. Archives municipales de Pornichet, Délibération du 8 février 1903. 
signaler et de demander qu'elles soient rectifiées ${ }^{27}$ ". Plusieurs autres communes bretonnes ont subventionné les éditions de ce guide (Saint-Brevin, Préfailles...). Au xx $\mathrm{x}^{\mathrm{e}}$ siècle, une hiérarchisation officieuse, validée par les guides, exploite la réputation des stations balnéaires. Les petites stations, plus modestes architecturalement et budgétairement, ne sont pas mises en avant comme les cités balnéaires dont la croissance dépend en grande partie de la saison estivale.

\section{L'élaboration d'un bréviaire touristique pour les sites balnéaires bretons}

Afin de pouvoir décrire significativement les sites autrement que par la seule affluence balnéaire, les guides touristiques vont introduire des images nouvelles, préparées par la redécouverte des provinces au cours de la première moitié du XIX ${ }^{\mathrm{e}}$ siècle et l'action des sociétés savantes et érudits locaux ${ }^{28}$, à partir d'une grille référentielle de lecture du territoire. Avant la Révolution de 1789, il n'existe pas d'image bretonne spécifique ${ }^{29}$. Cette partie ne fait évidemment pas le tour complet de la question, qu'il serait peut-être intéressant de faire à l'avenir, notamment pour éclairer les discours actuels des promoteurs touristiques.

\section{Le paysage à l'aune d'une maritimité de circonstance}

L'un des premiers arguments de différenciation des sites est aujourd'hui le paysage, c'est-à-dire l'étendue de pays qui s'offre à la vue. Le point de vue, à partir de la plage ou d'un point haut, est censé attirer le touriste. C'est un type de description adopté depuis au moins le début du $\mathrm{XIX}^{\mathrm{e}}$ siècle, dans tous les lieux touristiques qui attirent le voyageur, comme en Normandie étudié par François Guillet ${ }^{30}$. Le paysage apprécié apparaît comme un spectacle géographiquement limité. Les îles qui sont à portée permettent de fermer l'horizon. Déjà en 1863, Pol de Courcy, jouissant du panorama au sommet de la butte de Tumiac (presqu'île de Rhuys), évoque les plages du Croisic, les îles d'Houat, Hœdic et Belle-lle, la presqu'île de Quiberon $^{31}$. Le littoral breton, économiquement vivifié depuis le début du XIX ${ }^{\mathrm{e}}$ siècle par l'industrialisation de la pêche et le développement du commerce maritime, apporte aussi une grande animation appréciée des

27. VINCENT, Johan, L'Intrusion balnéaire; Les populations littorales bretonnes et vendéennes face au tourisme (1800-1945), Rennes, PUR, coll. " Histoire », 2007, p. 141-142.

28. Pour se rendre compte de l'action des sociétés savantes et des érudits locaux dans le secteur du tourisme, particulièrement au tournant des $\mathrm{XIX}^{\mathrm{e}}-\mathrm{XX}^{\mathrm{e}}$ siècles, on se reportera à l'ouvrage dirigé par Jean-Yves ANDRIEUX et Patrick HARISMENDY, Initiateurs et entrepreneurs culturels du tourisme (1850-1950), paru aux PUR en 2011.

29. BERTHO, Catherine, "L'invention de la Bretagne : genèse sociale d'un stéréotype ", Actes de la recherche en sciences sociales $\mathrm{n}^{\circ} 35,1980$, p. 46.

30. GuILlET, François, "Les guides consacrés à la Normandie pendant la première moitié du XIXe siècle (1915-1850)", dans CHABAUD, Gilles et al., op. cit., p. 510.

31. Pol DE COURCY, op. cit., p. 97. 
baigneurs. En face de la plage de Tréboul (aujourd'hui sur la commune de Douarnenez), "à très courte distance, est l'île Tristan, avec un îlot rocheux un peu plus loin; sur la droite, les falaises se déroulent bleuâtres et vaporeuses dans le bleu du ciel, enfermant la baie sillonnée de voiles blanches ${ }^{32}$ ". À la fin du siècle, à mesure qu'ils sont construits (la majorité des phares français actuels sont édifiés au cours du XIX ${ }^{\mathrm{e}}$ siècle), les phares font également partie de la description du panorama : Paul Joanne ${ }^{33}$ décrit la vue depuis le Mont Lénigo (Le Croisic), avec la baie, les îles de Hødic et de Houat, la côte morbihannaise, et le phare du plateau du Four, allumé en 1822 - une description assez semblable de celle d'Adolphe Joanne en 1867 mais plus circonscrite pour l'auteur du Guide Diamant de 1921, qui voit beaucoup moins loin (jusqu'à La Turballe et Guérande). En 1921, le phare du plateau du Four n'est plus visible que de la pointe du Croisic (à 2 kilomètres de là), peut-être dans l'objectif de faire faire le tour de la presqu'île au visiteur, ce qui était déjà possible en 1885 - en police de caractères plus petite.

Les points de vue sont déplacés à mesure que les stations balnéaires acquièrent du succès, avec une fixation des points de vue qui semble émerger au tournant des XIX ${ }^{\mathrm{e}}$ et $\mathrm{XX}^{\mathrm{e}}$ siècles $^{34}$. En 1867, Adolphe Joanne profite depuis Le Pouliguen du panorama sur l'entrée de la Loire et les îlots Petits et Grands-Charpentiers ${ }^{35}$; en 1921, ce panorama, enrichi du phare du Grand-Charpentier, allumé en 1888, est visible depuis la station concurrente de Pornichet ${ }^{36}$ (nous avons vu précédemment le glissement de l'affluence balnéaire dans la baie du Pouliguen) - du Pouliguen, le lecteur ne peut dorénavant plus voir grand-chose, la vue (très belle pourtant, selon ce guide) ne portant pas au-delà de la pointe de Chemoulin. Il est possible que les guides segmentent au début du $\mathrm{Xx}^{\mathrm{e}}$ siècle les panoramas par station balnéaire, réduisant ainsi le nombre d'éléments visibles. Précisons que, de la pointe de Chemoulin (Saint-Nazaire), la plus proche géographiquement (3 kilomètres) des éléments décrits, le phare est également visible selon le guide Diamant de 1921 mais c'est la seule chose que l'on voit de là-bas. L'horizon de La Baule, station en fond de baie, n'est pas bien orienté pour accorder ce point de vue; la station adopte d'autres stratégies. Les guides ne vont donc pas jusqu'à inventer des paysages.

32. RobIDA, A., La vieille France : Bretagne, Paris : La librairie illustrée, sans date (1895?), p. 211.

33. JoAnne, Paul, Bretagne, op. cit., p. 295.

34. La lecture de l'édition 2010-2011 du Petit Futé Loire-Atlantique semble toutefois montrer que les points de vue, moins systématiquement évoqués, ont encore légèrement varié au cours de la deuxième moitié du $\mathrm{xx}^{\mathrm{e}}$ siècle puisque la pointe de Chemoulin n'est plus indiquée.

35. JoAnne, Adolphe, Itinéraire général de la France..., op. cit., 1873, p. 532.

36. Anonyme, Guides Diamant : Nantes, Saint-Nazaire et les plages de la Loire-Inférieure, Paris, Hachette, 1921, p. 43-45. 


\section{Une omniprésence progressive du rocher}

Élément aujourd'hui reconnu comme indissociable du littoral breton, le rocher a longtemps été ignoré dans les descriptions des guides - le granite est le matériau le plus réputé mais il n'est pas omniprésent ${ }^{37}$. Michel Duval a été ainsi surpris de la faible place que Pol Potier de Courcy consacre, en 1863, à la description physique des côtes : il ne fait allusion ainsi à la mer que trois fois en 400 pages et préfère décrire villes et monuments ${ }^{38}$. La mer est pourtant appréciée depuis le XVIII ${ }^{\mathrm{e}}$ siècle, notamment pour son caractère tempétueux ${ }^{39}$. Les rochers (la falaise) ne sont alors qu'un obstacle, nécessaire au spectacle mais sans intérêt en tant que tel. Les populations locales semblent aussi peu sensibles à leur esthétique, même si leurs motivations pour parler du paysage dans les sources historiques ont pu biaiser leur regard. En 1872, le conseil municipal de Bréhat, soulignant la misère extrême de l'île, n'évoque que des rochers dénudés et des rivages appauvris ${ }^{40}$. Les premiers guides ne s'appesantissent pas sur ces éléments. Ainsi, si Adolphe Joanne indique en 1867 que « les flots viennent battre les rochers au pied du couvent ${ }^{41}$ " de Saint-Gildas-de-Rhuys, il s'intéresse plus à la mer qu'aux rochers. À noter que l'auteur se trompe et/ou trompe le lecteur car le couvent de Saint-Gildas-de-Rhuys se trouve dans le bourg de la commune, à quelques kilomètres du rivage (confusion avec un éventuel établissement de bains créé par les sœurs?). Cela n'empêche pas certains touristes d'apprécier l'esthétique de l'élément minéral dès le milieu du $\mathrm{XIX}^{\mathrm{e}}$ siècle, comme E. Bonnecarrière en 1856, qui décrit dans une lettre à Jules Michelet la Grande côte du Pouliguen, aux " rochers assez beaux ${ }^{42}$ ".

Le rocher ne devient un élément récurrent du discours touristique qu'à la fin du XIX ${ }^{\mathrm{e}}$ siècle. Depuis la seconde moitié du siècle, l'intérêt pour le rocher anthropomorphe est fort en Europe, dans l'art, la littérature symboliste, l'ethnologie, la linguistique ${ }^{43}$... Le sujet strictement minéral (où le rocher est l'objet de la représentation et non un élément de la composition) est travaillé par les peintres en Bretagne dans les années 1880 (par exemple, Emmanuel Lansyer, Ile d'Ouessant, 1885; Claude Monet, Les pyramides de Port-Coton, 1886; Henri Régnault, Rochers en Bretagne, 1886).

37. Michel, François, Les Côtes de France : paysages et géologie, Orléans, Éditions du BRGM, 1991.

38. Duval, Michel, "Bretagne et Bretons à travers quelques littérateurs et quelques guides du XIX ${ }^{\mathrm{e}}$ siècle ", dans CESBRON, Georges (dir.), Ouest et romantisme, Angers, Presses de l'Université d'Angers, 1991, p. 505.

39. CoRBIn, Alain, Le Territoire du vide; l'Occident et le désir de rivage (1750-1840), Paris, Aubier, coll. "Historique ", 1988.

40. Salomé, Karine, Les représentations des îles bretonnes (1750-1914); Études comparée du regard de l'autre et du regard sur soi, Thèse de doctorat en Histoire, université de Paris I-Panthéon Sorbonne, 2002, p. 489 (édition publiée : Rennes, PUR, 2003, 462 p.).

41. JoAnne, Adolphe, Itinéraire général de la France..., op. cit., 1867, p. 563.

42. MichelET, Jules, Correspondance générale, t. VIII, Paris, Librairie Honoré Champion, 1998, p. 131.

43. MARTin, Jean-Hubert (dir.), Une Image peut en cacher une autre (Arcimboldo, Dalí, Raetz), Paris, Éditions de la Réunion des Musées Nationaux, 2009, p. 180. 
Les guides s'en emparent alors. En 1892, Constant de Tours s'attarde sur les roches de Pléneuf, les " amas de rochers pittoresques, chaos étranges " de Portrieux, les "rochers semés à profusion " de Brignogan ${ }^{44}$. Il modère ce " cliché " pour les côtes de l'Atlantique, "moins sauvages et déchiquetées que sur le littoral de la Manche ${ }^{45}$ ". Toutefois, dès que cela est possible, il n'hésite pas à citer les rochers à proximité des plages du Pouliguen, du Croisic, de Saint-Pierre-Quiberon, de Quiberon... En 1907, Richard évoque au Croisic des " rochers étranges, crevassés par les assauts de la mer ", sur la Grande Côte ${ }^{46}$. La nouvelle norme esthétique est également assimilée par les populations locales, comme à Bréhat où le conseil municipal signale en 1899 le côté pittoresque de l'île. Pour l'historienne Karine Salomé, c'est à la fin du XIX ${ }^{\mathrm{e}}$ siècle que les insulaires s'approprient la grille de lecture du paysage élaborée par les visiteurs étrangers à l'île ${ }^{47}$. L'assimilation est donc particulièrement rapide et, dès 1930, Maurice Le Lannou déclare que "les rochers de la côte bretonne sont peut-être la cause la plus importante de sa fortune touristique ${ }^{48}$ ".

\section{L'air vivifiant, apport salvateur permanent des côtes bretonnes}

Le guide doit également motiver les touristes en présentant des sites maritimes où le corps et l'esprit se régénèrent. Les auteurs profitent de la promotion du bain de mer comme pratique médicale au XIX ${ }^{\mathrm{e}}$ siècle, confortant ainsi les bienfaits d'un séjour sur le littoral. "En dehors des bains, l'air de la mer offre pour certains une véritable cure. [...] Il provoque [aussi] des crises radicales que les plus habiles médecins avaient en vain demandées aux sudorifiques de la pharmacie ${ }^{49}$. " Bertall explique la frénésie pour les bains de mer : "C'est au bord de la mer, en respirant l'air salin, en s'assimilant ses fortifiants effluves, que l'on peut arriver à vaincre l'énervement produit par cette fièvre de la vie moderne ${ }^{50}$. "Ce type de discours persiste dans les années 1920. J. Rouch prévient le touriste qu'il doit se préoccuper du climat de la station balnéaire qu'il a choisie : les caractères météorologiques peuvent " ne pas toujours convenir à certains tempéraments ${ }^{51} "$.

44. Constant DE Tours, Vingt jours en Bretagne : de Saint-Malo à Brest, Paris, Librairiesimprimeries réunies, 1892 , p. 33, p. 55 et p. 126.

45. Constant DE Tours, Côtes bretonnes de Nantes à Brest et Basse-Loire, Paris, May et Motteroz, vers 1900, p. 33.

46. RICHARD, Guide du voyageur en France; Réseau de l'Ouest, Paris, Hachette, coll. "Guides Joanne ", 1907, p. 29.

47. SAlomé, Karine, op. cit., p. 489.

48. LE LANNOU, Maurice, Itinéraires de Bretagne; Guide géographique et touristique, Paris, J. B. Baillière et fils éditeurs, sans date (années 1930), p. 9.

49. ANONYME, Album de la côte guérandaise et ses environs; Quelques renseignements pour les excursionnistes de la Presqu'̂̀le Guérandaise, 1907, p. 15.

50. Bertall, Les Plages de France, Paris, Marpon et Flammarion, 1880, p. 1.

51. Rouch, J., Pour comprendre la mer, à l'usage des touristes, Paris : Hachette, 1923, p. 1. 
Or, en Bretagne, la présence de l'océan atlantique et de la Manche permet des bains revigorants. De plus, le baigneur évite la pollution des mers fermées et surchauffées, les guides ignorant facilement les usines de conserves et les entreprises goémonières en fort développement au cours du XIX siècle. Port-Navalo devient " le plus beau joyau des petits ports de cet archipel, où ce grand Océan se plaît à verser ses eaux les plus limpides, les plus pures, et qui, par son adorable baie et son aspect aussi séduisant que pittoresque, charme la vue, l'esprit et le cœur ${ }^{52}$ ». E. Le Gal explique, dans son guide du touriste en presqu'îles de Rhuys et de Locmariaquer, que "l'hiver, de continuelles tempêtes déchaînent leurs violences sur ces rivages; l'été, le ciel est clément et hospitalier, le climat salubre, l'air imprégné d'émanations balsamiques et marines ${ }^{53}$ ". Dans l'Entre-deux-guerres, l'air vivifiant (mais plus l'eau) est un argument touristique, contrebalançant les publicités de la Côte d'Azur vantant le soleil nécessaire à la mode récente du bronzage. Maurice Le Lannou constate que " les brochures de propagande qui vous invitent à venir faire en Bretagne "une cure d'air iodé" n'ont pas tort ${ }^{54}$ ".

Le vent reste toutefois un défaut pour le villégiateur. Le géographe Pierre Escourrou a démontré que, parmi les choix d'implantation d'une station balnéaire, l'abri du vent est essentiel ${ }^{55}$. Le guide régional Michelin, dans son édition de 1928, précise quelles plages sont abritées du vent ${ }^{56}$. Si la station balnéaire est déjà fréquentée, le guide sait néanmoins transcender cette imperfection. En 1879, Adolphe Joanne adapte son discours pour Préfailles, lieu de bains fréquenté : «La plage, très exiguë, très caillouteuse, mais jolie, se trouve au fond d'une petite anse; le vent du Sud-Ouest y souffle avec violence; mais la mer, quoique souvent agitée, y est toujours d'une limpidité parfaite ${ }^{57}$. » Paul Joanne, dans ses guides de 1900 et de 1904, rajoute même une qualité due au vent : "Le vent du Sud-Ouest y souffle avec violence (très belles vagues) ${ }^{58}$. " Les guides ne condamnent pas la station balnéaire qui a du succès. Paul Gruyer observe qu'à Quiberon, "le pays est dénudé et exposé à tous les vents, mais la mer est magnifique ${ }^{59}$ ". Le guide propose des compensations quand le site (c'est-à-dire, en creux, la station) est digne d'intérêt.

52. Ledivellec, J. M., La Presqu'île de Rhuys en Bretagne ou le canton de Sarzeau; Guide des baigneurs et des touristes, Vannes, Imprimerie Galles, 1864, p. 2-3.

53. LE GAL, E., Guide du touriste : Rhuys, Locmariaquer, Gavr-inis, questions archéologiques, Vannes, Imprimerie Galles, 1879, p. 1.

54. Le Lannou, Maurice, op. cit., p. 9.

55. Escourrou, Pierre, Climat et tourisme sur les côtes françaises de Dinard à Biarritz, Thèse de doctorat en Géographie, Université de Paris I-Panthéon Sorbonne, 1980, p. 142.

56. Anonyme, La Bretagne; Guide régional Michelin, Clermont-Ferrand, Michelin et $\mathrm{C}^{\mathrm{ie}}$, 1928.

57. JoAnne, Adolphe, Itinéraire général de la France : De la Loire à la Gironde, Paris, Hachette, coll. "Guides Joanne », 1879, p. 416.

58. JoAnne, Paul, Itinéraire général de la France : La Loire..., op. cit., p. 333 et Paul Joanne, Itinéraire général de la France : De la Loire aux Pyrénées, Paris, Hachette, coll. " Guides Joanne ", 1904, p. 6.

59. GruYer, Paul, Bretagne, Paris, Hachette, coll. " Guides Joanne », 1914, p. 457. 


\section{L'impact des guides dans l'aménagement balnéaire}

Eux-mêmes preuves d'une certaine reconnaissance, les guides se font la caisse de résonance du succès balnéaire. Ils introduisent un cercle vertueux (ou vicieux) au cours du $\mathrm{xx}^{\mathrm{e}}$ siècle, en s'appuyant sur une grille de lecture de la région bretonne précédemment élaborée : plus la station balnéaire s'accorde avec le modèle, plus elle bénéficie de l'attention des guides.

\section{Création d'une nature humanisée}

L'adoption de référentiels par les guides peut être une influence des nouvelles formes du texte littéraire qui émergent au XIX ${ }^{\mathrm{e}}$ siècle : la pratique fragmentaire. Laurent Matthey observe que cette pratique, développée par les romantiques et radicalisée au tournant des $\mathrm{XIX}^{\mathrm{e}}-\mathrm{XX}{ }^{\mathrm{e}}$ siècles, mêle sites et impressions, en multipliant les points de vue. Lieux et effusions sont synthétisés dans la forme totale du paysage, produisant une forme de territoire qui se matérialise à la même époque : le jardin paysager ${ }^{60}$. Le discours touristique distord la réalité du site, par exemple avec la grille de lecture évoquée dans la partie précédente. En même temps, les guides sont prescripteurs. À partir de l'exemple de Sable-d'Or-les-Pins, Roland Vidal, reprenant les termes du philosophe Alain Roger, explique qu'à une articulation in visu (avec la production d'images) s'ajoute une articulation in situ (avec le projet d'une station paysagère à la fin des années 1920) : l'esthétique du lieu est mise en valeur par la manière dont on l'aménage ${ }^{61}$. La création des jardins à partir de la seconde moitié du XIX ${ }^{\mathrm{e}}$ siècle encourage ainsi la reconnaissance favorable du site et les pratiques, ce que les guides répercutent dans leurs propos. Aux abords de Saint-Lunaire, Richard constate que « les bois de Pontual et le parc de l'ancien château de ce nom, remplacé par la villa Revault, ainsi que la pointe et la grotte de l'Hirondelle, offrent d'agréables buts de promenade ${ }^{62}$ ".

Afin d'accorder aux touristes des sites qui leur conviennent, le site « primitif " disparaît physiquement sous les nouveaux atours de la station balnéaire. Déjà en 1881, L. Lagneau observe qu'à Dinard, " l'ancien aspect sauvage se révèle encore, mais au lieu d'herbes marines et de goëmons, ce ne sont plus que jardins suspendus où poussent en pleine terre le figuier, l'aloès, la citronnelle, les camélias, le myrte, le jasmin et même le palmier ${ }^{63}$ ". Dans le même temps, les baigneurs détournent à leur profit des investissements

60. MATTHEY, Laurent, "Quand la forme témoigne : réflexions autour du statut du texte littéraire en géographie ", Cahiers de géographie du Québec, décembre 2008, p. 413.

61. VIDAL, Roland, La Construction paysagère d'une identité territoriale; Imaginaire et réalité dans une station balnéaire des Côtes-d'Armor : Sables-d'Or-les-Pins, Thèse de doctorat en Sciences de l'environnement, École Nationale du Génie rural, des Eaux et des Forêts, 2003, p. 14.

62. RICHARD, op. cit., p. 68.

63. Lagneau, L., Dinard et ses environs : guide du casino, curiosités, promenades, excursions et tous les renseignements à l'usage des baigneurs et des touristes, Rennes, Imprimerie Oberthur et fils, 1881, p. 13-14. 
réalisés prioritairement pour les populations littorales. La fixation des dunes ordonnée en 1810 conduit à la plantation de pins sur le littoral. Ces bois sont ensuite empruntés par les baigneurs, qui parfois y construisent leur villa. Le boisement de La Baule, exécuté à partir de 1818 mais réalisé essentiellement à la fin du siècle, sera ainsi un atout majeur pour la station balnéaire. Au début du $\mathrm{Xx}^{\mathrm{e}}$ siècle, La Baule, devenue " une station forestière autant que maritime", est surnommée l'Arcachon de Bretagne ${ }^{64}$. Jacques Levron se permet un audacieux raisonnement (faux, au demeurant), quand il écrit que l'implantation du chemin de fer en 1879 a conduit à « la transformation du désert en une cité balnéaire, la plus aimable et la plus florissante " mais c'est une évolution qui apparaît logique dans cet esprit. Il poursuit : " dernière création, la Baule-les-Pins offre la parfaite image de la nature humanisée. [...] Et du moins La Baule a gardé ses bois de pins qui lui confèrent un cachet rare $^{65}$. " La nature a revêtu une seconde nature - l'œuvre de l'homme - que les guides mettent alors en avant.

\section{Adaptation du site à la modernité}

L'occultation des impuretés primitives, par rapport au modèle, s'accompagne du mariage de deux paradigmes apparemment contraires : la station balnéaire doit devenir " une ville moderne de campagne ${ }^{66}$ ". Le discours hygiéniste enjoint la maîtrise du territoire. Dans les guides de la première moitié du $\mathrm{xx}^{\mathrm{e}}$ siècle, les villes récentes ou créées ex-nihilo sont ainsi mises en avant. Ainsi, Quiberon, " ville nouvelle, jouit de tous les avantages du progrès : [...] les rues sont larges, bien tracées; un magnifique boulevard longe la plage, sur laquelle les enfants s'ébattent à leur aise ${ }^{67}$ ". La Baule illustre les exigences modernes adaptées directement sur le terrain. La clientèle bauloise, devenue à partir de l'Entre-deux-guerres bourgeoise et mondaine, réclame les dernières modernités.

"Les villégiateurs sont tous agréablement impressionnés par l'aspect général de propreté des rues et des avenues de La Baule. Les villas, les hôtels, les magasins plaisent par leur bon goût de leur construction, la variété et l'ingéniosité des étalages et des vitrines. Les différentes artères de la station, grâce à un excellent entretien, se prêtent aisément à l'intense circulation saisonnière ${ }^{68}$."

Cet hygiénisme poussé à l'extrême chagrine certains auteurs : le côté propret dénature le site. " On peut déplorer ces allées trop peignées, cet acclimatement des fleurs des serres chaude : camélias, mimosas, bégonias,

64. Monmarché, Marcel, Itinéraire général de la France : La Loire, Paris, Hachette, coll. "Guides Joanne ", 1908, p. 362-363.

65. LeVron, Jacques, La Haute-Bretagne, Grenoble, B. Arthaud, 1938, p. 59-61.

66. ROUILlaRd, Dominique, op. cit., p. 174.

67. Anonyme, Thermes et plages; La Bretagne, guide du touriste au bord de mer, Paris, Guides illustrés, 1912, p. 69.

68. Anonyme, La Plage du Soleil : La Baule, La Baule, Syndicat d'initiatives de la Côte d'Amour et de la presqu'île guérandaise, 1936, p. 32. 
plantes méridionales qui, grâce à la merveilleuse exposition de la Baule, s'épanouissent comme sur la Côte d'Azur. Mais ce reproche n'est-il pas mérité par toutes les plages à la mode ${ }^{69}$ ? " Les stations balnéaires qui réussissent le mariage entre la ville et la campagne sont celles qui profitent de la plus large audience dans les guides. C'est ainsi qu'un cercle touristiquement vertueux se forme. Le discours référencé construit dans la seconde moitié du XIX ${ }^{\mathrm{e}}$ siècle permet de maquiller cette réalité.

Les guides répondent finalement à une demande sociale, qu'ils doivent anticiper. Ils déconseillent certains sites dont l'usage ne conviendrait pas aux baigneurs, c'est-à-dire qui s'écartent du modèle pressenti. Pour le guide Bædeker, Concarneau, qui possède plusieurs petites plages, est " une station balnéaire peu recommandable à cause de l'odeur infecte que répand le port à marée basse, augmentée encore de celle qui s'échappe des sardineries ${ }^{70}$ ". Si les conserveries sont visitées, si les ports sont pour les touristes un spectacle, l'activité de pêche (par exemple) ne s'accorde pas avec la villégiature : toute autre activité que balnéaire est mise à distance, pouvant être vue mais quand cela est utile. La Turballe est bannie par les guides jusqu'à la Première Guerre mondiale : " important port de pêche sardinier, sale et populeux ", " il y a quelques baigneurs pendant l'été [...], mais le voisinage des sardineries aux odeurs d'huile et de friture éloigne la foule ${ }^{71}$ ". L'évocation de ces désagréments valide un discours. Quiberon, "pittoresque port et importantes sardineries ${ }^{72}$ ", ne connaît pas ces critiques. Or cette dichotomie du traitement est possible parce que La Turballe n'est pas encore une station balnéaire, ce qu'elle deviendra dans l'Entre-deux-guerres; Quiberon l'est depuis la fin du XIX ${ }^{\mathrm{e}}$ siècle. La dernière référence négative pour La Turballe (" très sale ") a été relevée dans le Guide Diamant : Bretagne de $1918{ }^{73}$. L'évacuation descriptive de ce qui est estimé comme une pollution est reproduite par les caméramans amateurs des années 1920 et 1930, résumant le littoral breton à un réservoir d'un temps lissé par une bourgeoisie incapable de rendre compte de ses aspérités, dédié uniquement au bonheur ou à la méditation ${ }^{74}$.

Il serait injuste de considérer que les auteurs de guide peuvent rendre totalement idéal un site, quel qu'il soit. Leur rôle n'en reste pas moins important : ils renforcent les atouts des destinations, de cette manière encouragées à être mieux mises en valeur localement, et éventuellement réduisent l'impact de leurs défauts. Dans un premier temps, jusqu'au moins la première massi-

69. LeVRON, Jacques, op. cit., p. 61.

70. BÆDEKER, Karl, Le Nord-Ouest de la France, de la frontière belge à la Loire, Leipzig, Karl Bædeker éditeur, 1902, p. 377.

71. AnONYME, Bains de mer de Bretagne..., op. cit., p. 119.

72. Anonyme, Bretagne, côte sud, Chemin de fer de Paris à Orléans, 1928, p. 8.

73. Anonyme, Guides Diamant : Bretagne, Paris, Hachette, 1918, p. 157.

74. LE GALL, Laurent, "Le temps recomposé; Films amateurs et sociétés littorales dans la Bretagne des années 1920 et 1930 ", Annales de Bretagne et des Pays de l'Ouest, septembre 2010, p. 141-144. 
fication touristique (tournant $\mathrm{XIX}^{\mathrm{e}}-\mathrm{xX}^{\mathrm{e}}$ siècles), les éditeurs ont dû cerner les dynamiques territoriales, pour construire un discours qui a été rapidement repris par les populations locales. Les effets d'entraînement ont survalorisé les territoires dynamiques, qui ont dû, au xx ${ }^{\mathrm{e}}$ siècle, s'adapter, ou adapter les guides (selon les situations), pour continuer de profiter de ces mannes touristiques. Pour Hélène Hatzfeld, décortiquant la demande actuelle, le site remarquable est celui qui ajoute des valeurs esthétiques aux valeurs d'ancrage historique $^{75}$. Le guide y contribue pleinement. Le passage au modèle économique payant des guides nationaux, en 1920 pour le guide Michelin par exemple ${ }^{76}$, peut avoir également renforcé l'exigence des lecteurs, désireux de découvrir, ironiquement, de "vrais " sites touristiques. Les communes littorales où l'activité balnéaire est marginale, voire nulle, peuvent faire l'objet de critiques, ce qui, d'une certaine manière, permet de mettre à nouveau en avant les autres espaces décrits, sans troubles pour les affaires des éditeurs de guide. En élaborant une grille de lecture des sites, en " régionalisant " la destination, le guide permet une assimilation plus importante du discours.

Malgré un recopiage partiel d'une édition à l'autre, le discours n'est pas figé : le guide relaie la perception et non la réalité du site. Marc Francon, à partir du Guide Vert Michelin, a déjà constaté le recyclage des informations et la normalisation affichée de la curiosité, propices à l'ignorance de sites ou monuments qui seront, plus tardivement, déclarés intéressants ${ }^{77}$. C'est la perception qui entraîne la découverte de sites, selon le même principe de la validation (par l'affluence, par les "impératifs souhaités " par les touristes). Le sens commun, la tradition, une sorte de consensus culturel, évoqués par Marc Francon, ne sont pas les seuls référents, sauf dans le discours de présentation de l'initiative, pour des raisons morales - ces référents sont moins attaquables que l'affluence pour l'affluence. La mécanique de la distinction, découverte par Thorstein Veblen, est un moteur puissant dans le tourisme, notamment pour des questions d'image. Elle s'insère dans de nouveaux projets, dans de nouvelles pratiques, qui amènent des perceptions différentes du site et donc une grille supplémentaire de lecture, sans toujours faire table rase des précédentes références : par exemple, à partir de l'Entre-deux-guerres, le retour à la nature (camping, naturisme...) en Europe nécessite des adaptations aux usages jusqu'alors inconnus et dont les guides s'empareront, essentiellement après la guerre. Ils sont à la fois l'objet d'un déterminisme conservateur et l'un des meilleurs médias de l'innovation, quand elle est socialement acceptée : le tourisme est fait d'ambivalences...

75. HATZFELD, Hélène, "À la recherche d'un bien commun : la demande de paysage ", dans : MARCel, Odile, dir., Paysages modes d'emploi; Pour une histoire des cultures de l'aménagement, Seyssel, Éditions Champ Vallon, coll. « Les cahiers de la compagnie du paysage ", 2006, p. 281.

76. FRANCON, Marc, op. cit., p. 5. À ma connaissance, il n'existe aucune étude scientifique sur la variabilité de l'exigence du consommateur envers un produit, selon que celui-ci soit payant ou gratuit.

77. Ibidem, p. 46 et p. 48-49. 